\title{
Lower occlusion pressure during resistance exercise with blood-flow restriction promotes lower pain and perception of exercise compared to higher occlusion pressure when the total training volume is equalized
}

\author{
SD Soligon ${ }^{1}$, ME Lixandrão ${ }^{2}$ TMPC Biazon ${ }^{1}$, V Angleri $^{1}$, H Roschel $^{2}$, CA Libardi $^{1}$ \\ ${ }^{1}$ MUSCULAB - Laboratory of Neuromuscular Adaptations to Resistance Training, Department of Physical \\ Education, Federal University of São Carlos - UFSCar, São Carlos, Brazil \\ ${ }^{2}$ School of Physical Education and Sport, University of São Paulo - USP, São Paulo, Brazil
}

\author{
Received: September 27, 2017
}

Accepted: August 21, 2018

\begin{abstract}
Low-intensity resistance exercise with blood-flow restriction (BFR) promotes similar adaptations to high-intensity resistance exercise (HI-RE). Interestingly, BFR has been demonstrated to be effective for a wide range of occlusion pressures. However, the occlusion pressure magnitude may alter the psychophysiological stress related to BFR as measured by rating of perceived exertion scale (RPE) and rating of pain. We aimed to compare the RPE and pain levels across different magnitudes of occlusion pressures, promoting new knowledge regarding occlusion pressure on stress related to BFR. All BFR protocols ranging between $40 \%$ and $80 \%$ of total arterial occlusion (BFR40, BFR50, BFR60, BFR70, and BFR80) were compared to HI-RE in 12 participants using a randomized and crossover design $72 \mathrm{~h}$ apart. BFR protocols and HI-RE were performed with $30 \%$ and $80 \%$ of one-repetition maximum (1RM) test value, respectively. RPE and pain levels were measured before exercise and immediately after each set. BFR protocols (i.e., BFR40 and BFR50) presented overall lower RPE response compared to higher-pressure BFR (i.e., BFR70 and BFR80) and HI-RE conditions. For pain levels, low-pressure BFRs (i.e., BFR40 and BFR50), and HI-RE showed lower values than high-pressure BFR protocols (i.e., BFR60, BFR70, and BFR80). In conclusion, low-pressure BFR protocols promote lower RPE and pain compared to high-pressure BFR protocols (between $60 \%$ and $80 \%$ of occlusion pressure), when total training volume (TTV) is equalized. In addition, HI-RE promotes similar levels of pain, but higher RPE than low-pressure BFR, probably due to the higher TTV.
\end{abstract}

Keywords: resistance training, blood-flow restriction, psychophysiological stress, rating of perceived exertion, pain

\section{Introduction}

High-intensity resistance exercise [HI-RE; $>65 \%$ one-repetition maximum (1RM)] is typically recommended as the most effective training protocol to promote muscle mass accrual (i.e., muscle hypertrophy) $(2,3)$. However, recent evidence suggests that low-load resistance exercise (RE; 20\%-40\% 1RM) associated with partial blood-flow restriction (BFR) may result in similar increases in muscle mass compared to $\operatorname{HI}-\mathrm{RE}(12,13,26,32,33)$. Interestingly, BFR has been demonstrated to be effective for a wide range of occlusion pressures (14). Indeed, a recent meta-analysis carried out by our laboratory demonstrated

Corresponding author: Cleiton Augusto Libardi, PhD

MUSCULAB - Laboratory of Neuromuscular Adaptations to Resistance Training, Department of Physical Education Federal University of São Carlos - UFSCar, Rod. Washington Luiz, km 235

SP-310, CEP 13565-905, São Carlos, SP, Brazil

Phone/Fax: +55 163306 6777; E-mail: c.libardi@ufscar.br 
similar increases in muscle mass between HI-RE and distinct BFR protocols, even with far different relative occlusion pressures $(6,12-15,28)$.

Although it seems not to influence muscle mass accrual, occlusion pressure seems to affect the psychophysiological stress related to BFR as measured by rating of perceived exertion (RPE) scales $(1,25)$. For instance, adding BFR to low-load RE resulted in increased RPE response when compared to the same protocol without BFR (22). Moreover, higher occlusion pressures are associated with higher RPE levels (18). Despite this, recently, Loenneke et al. (19) investigated the dose response of occlusion pressure (i.e., $40 \%, 50 \%$, $60 \%, 70 \%, 80 \%$, and $90 \%$ ) on RPE levels and showed no significant response. Importantly, total training volume [TTV; sets $\times$ repetitions $\times$ load $(\mathrm{kg})]$ was not equalized between protocols. TTV has been shown to influence RPE response, with higher TTV being associated with elevated RPE response (8). Therefore, to properly understand the effects of occlusion pressure manipulations on RPE response, it seems imperative to equate TTV across BFR protocols.

Another important measure of BFR-related stress is the level of pain (16, 29, 30). Studies comparing the levels of pain between protocols with and without BFR have yielded contrasting results. Some studies showed higher pain levels for BFR protocols $(16,29)$ and others showed no significant differences (30). These different findings may be, at least, partially explained by discrepancies between studies (e.g., cuff type and width and occlusion pressure). In addition, data are still lacking with regard to the understanding of the effects of occlusion pressure manipulation on pain levels.

Therefore, this study aimed to investigate the effects of TTV-equated BFR protocols with different occlusion pressures on RPE and ratings of pain. In addition, all BFR protocols were compared with conventional HI-RE.

\section{Methods}

\section{Participants}

Twelve male subjects (age: $24.5 \pm 1.5$ years, height: $1.78 \pm 0.04 \mathrm{~m}$, body mass: $83.4 \pm$ $11.2 \mathrm{~kg}$ ) participated in this study. Subjects were not engaged in any kind of regular RE and/ or aerobic training for at least 12 months prior to the experimental period, were free from any musculoskeletal disorders, and had not used drugs or medications that could affect physical performance. In addition, subjects were excluded if they had presented more than one risk factor for venous thromboembolism, obesity $\left(\mathrm{BMI}>30 \mathrm{~kg} / \mathrm{m}^{2}\right)$, cardiac dysrhythmia, and/or cardiac failure. The study was approved by the Ethics Committee of the local University (no. 466/12). Subjects gave their informed written consent prior to their inclusion into the study.

\section{Experimental design}

During the first visit, participants completed an anamnesis, anthropometric measurements, and were familiarized with the knee extension exercise. Twenty-four hours later, participants performed two maximum dynamic muscle strength tests (1RM), with 72-h rest apart. If 1RM values differed more than 5\% from previous test, a subsequent test was performed after $72 \mathrm{~h}$. Later, participants were familiarized with the BFR protocols. Seventy-two hours later, participants performed the following RE protocols in a randomized manner with 72-h rest intervals between them: (1) BFR at $40 \%$ of occlusion pressure (BFR40), (2) BFR at $50 \%$ of occlusion pressure (BFR50), (3) BFR at $60 \%$ of occlusion pressure (BFR60), (4) BFR at $70 \%$ 
of occlusion pressure (BFR70), (5) BFR at 80\% of occlusion pressure (BFR80), and (6) HI-RE. In order to prevent or at least minimize an interference effect, a "randomized protocol orders of Williams design" (Latin square) (11) was used. Williams' crossover design attempts to balance any carryover effects. The TTV was equated between all BFR conditions. RPE and pain levels were measured after each exercise set and immediately after the final set.

\section{Rating of perceived exertion (RPE)}

It was measured immediately after each set of RE training protocols through the standard 6-20 Borg scale, similar to that used by Row et al. (24). Subjects were instructed on how to respond to RPE before each exercise visit according to the following information: "We want you to tell us your perception of effort, that is, how difficult and strenuous you feel this exercise. Perception of effort depends primarily on the tension and fatigue in your muscles. We want you to respond using a 6-20 scale, where 6 means 'no effort' and 20 means 'maximum effort.' Any question?" Subjects confirmed that they fully understood how to respond to the RPE scale before beginning the RE protocol.

\section{Ratings of pain}

In a similar manner to RPE assessment, pain levels were evaluated after each set during the RE protocols using a visual analog scale (VAS). Similarly, participants were instructed on how to respond to the VAS. The value of 10 was classified as: "the worst pain of lactic acid that you have experienced in your quadriceps." This method has been previously used by Assumpção et al. (4).

\section{Maximum Dynamic Strength Test}

Bilateral quadriceps maximum dynamic strength was measured by the $1 \mathrm{RM}$ test on a knee extension machine (NKR Effort; Nakagym, São Paulo, Brazil) following previous recommendation (5). Subjects performed an initial general warm-up on a bicycle ergometer (Ergo 167 Cycle, Ergo Fit, Pirmasens, Germany) at $20 \mathrm{~km} / \mathrm{h}$. This was followed by a specific warmup consisting of one set of eight repetitions at $50 \%$ of the estimated $1 \mathrm{RM}$, followed by one set of three repetitions at $70 \%$ of the estimated $1 \mathrm{RM}$ with a 2 -min-rest interval between sets. Three minutes after the warm-up, subjects had about five attempts to achieve their 1RM. The smallest incremental load for the next attempt was approximately $1 \mathrm{~kg}$. A 3-min interval was allotted between the attempts and the highest load achieved (full eccentric-concentric movement with $90^{\circ}$ range of motion) was considered as $1 \mathrm{RM}$.

\section{Determination of arterial occlusion pressure}

Participants were requested to rest comfortably in supine position - for approximately $15 \mathrm{~min}$ - while a standard blood pressure cuff (i.e., static pressure control) $(17.5 \mathrm{~cm} \times$ 94 cm - JPG; Industria Comercio Assistência técnica Mat Hospitalar Ltda Me, São Paulo, Brazil) was positioned on the inguinal fold region of the thigh. Then, a vascular Doppler probe (DV-600, Martec, Ribeirão Preto, Brazil) was placed over the tibial artery to capture its auscultatory pulse. To determine complete BFR (pulse elimination pressure), the pressure cuff was inflated up to the point at which the auscultatory pulse was interrupted (9). For visual details on the procedures for the determination of BFR pressure and administration of BFR-RE, please refer to previous detailed description in the study of Gualano et al. (9). Complete BFR was individually measured for each leg of the same participant and reassessed at every training session. 


\section{Familiarization to BFR protocol}

Subjects warmed up for $5 \mathrm{~min}$ in a bicycle ergometer $(25 \mathrm{~W}, 20-25 \mathrm{~km} / \mathrm{h})$. Then, they performed a warm-up on the knee extension machine (NKR Effort) that consisted of one set of 10 repetitions at 50\% 1RM. Three-minutes later, subjects performed three sets of 15 repetitions at $30 \%$ of 1 RM, with 1 -min rest intervals between sets. Each one of the three sets was performed at different percentages of complete BFR $(40 \%, 60 \%$, and $80 \%$, respectively). Occlusion pressure was held constant during rest intervals, with values used in the previous set. Five seconds after the beginning of the subsequent set, the pressure was adjusted for the new set. Immediately after the end of each set, subjects were asked to rate their RPE (24) and pain levels (4).

\section{RE protocols}

RE protocols were performed in a bilateral knee extension machine (NKR Effort). Subjects warmed up for 5-min in a bicycle ergometer with a load of $25 \mathrm{~W}$ at $20-25 \mathrm{~km} / \mathrm{h}$, followed by a specific warm-up in the knee extension machine consisting of one set of 10 repetitions at $50 \%$ of 1RM. BFR protocol (same cuff used on "determination of arterial occlusion pressure" topic) consisted of three sets of 15 repetitions at $30 \%$ of $1 \mathrm{RM}$ with occlusion pressures ranging from $40 \%$ to $80 \%$ of total BFR (Table I). The HI-RE protocol consisted of three sets of 10 repetitions at $80 \%$ of $1 \mathrm{RM}$ without BFR. For all BFR protocols, the occlusion pressures were maintained throughout the exercise session. The rest interval between the sets was set at $1 \mathrm{~min}$. The movement speed was set at approximately $1 \mathrm{~s}$ for concentric and eccentric phases, standardized by a metronome.

\section{Statistical analyses}

Data were first inspected for normality by the Shapiro-Wilk's test. One-way repeated measures analysis of variance was performed to compare the mean RPE and pain for all sets (average of all sets), and TTV [sets $\times$ repetitions $\times$ load $(\mathrm{kg})$ ] between protocols. In case of significant $F$ values, a Tukey's adjustment was used for multiple comparison purposes. The significance level was set at $p<0.05$. Data are presented as mean \pm standard deviation.

Table I. Resistance exercise protocols

\begin{tabular}{|l|c|c|c|c|c|c|c|}
\hline Protocols & $\begin{array}{c}\text { 1RM } \\
(\%)\end{array}$ & $\begin{array}{c}\text { BFR } \\
\text { pressure } \\
(\%)\end{array}$ & $\begin{array}{c}\text { BFR } \\
\text { pressure } \\
(\mathbf{m m H g})\end{array}$ & $\begin{array}{c}\text { Intervals } \\
\mathbf{( s )}\end{array}$ & Sets & Repetitions & $\begin{array}{c}\text { TTV } \\
\text { (kg) }\end{array}$ \\
\hline BFR40 & 30 & 40 & $57.0 \pm 4.5$ & 60 & 3 & 15 & $1,731.4 \pm 255.6$ \\
\hline BFR50 & 30 & 50 & $72.9 \pm 5.0$ & 60 & 3 & 15 & $1,731.4 \pm 255.6$ \\
\hline BFR60 & 30 & 60 & $88.0 \pm 7.5$ & 60 & 3 & 15 & $1,731.4 \pm 255.6$ \\
\hline BFR70 & 30 & 70 & $100.3 \pm 9.6$ & 60 & 3 & 15 & $1,731.4 \pm 255.6$ \\
\hline BFR80 & 30 & 80 & $117.8 \pm 8.7$ & 60 & 3 & 15 & $1,731.4 \pm 255.6$ \\
\hline HI-RE & 80 & 0 & 0 & 60 & 3 & 10 & $3,078.0 \pm 454.3 *$ \\
\hline
\end{tabular}

HI-RE: high-intensity resistance exercise; BFR: blood-flow restriction; 1RM: one-repetition maximum test; BFR pressure (\%): blood-flow restriction pressure (\%) delta percentage of total occlusion pressure; TTV: total training volume. $* p<0.05$ when compared to all BFR protocols 


\section{Results}

Total training volume (TTV)

HI-RE protocol showed significantly greater TTV values compared to all other protocols performed with BFR $(p<0.0001)$. All repetitions of each set were completed by all the participants.

\section{Rating of perceived exertion (RPE)}

RPE showed higher values for BFR70, BFR80, and HI-RE compared to BFR40 ( $p=0.002$, $p<0.0001, p=0.002$, respectively). Similarly, RPE showed higher values for BFR70, BFR80, and HI-RE compared to BFR50 ( $p=0.005, p<0.001, p=0.005$, respectively). In addition, BFR80 and HI-RE also showed significantly higher RPE values compared to BFR50 ( $p<0.0001$ and $p=0.0052$, respectively) (Fig. 1A).

\section{Ratings of pain}

The BFR60, BFR70, and BFR80 protocols showed higher ratings of pain compared to BFR40 ( $p=0.03, p=0.008, p=0.0002$, respectively), BFR50 ( $p=0.0249, p=0.0120$, $p=0.0004$, respectively), and HI-RE ( $p<0.0001$; Fig. 1B).

\section{Discussion}

This study aimed to investigate the effects of BFR with different occlusion pressures with equated TTV on RPE and ratings of pain. A secondary purpose was to compare BFR protocols with a conventional HI-RE. The main results showed that lower-pressure BFR protocols generally promoted lower RPE and pain levels compared to higher-pressure BFR protocols. In addition, HI-RE protocol showed higher RPE than lower-pressure BFR protocols.

Previous studies have investigated the effects of occlusion pressure manipulation during BFR on RPE levels $(18,19,27,31)$. For instance, Loenneke et al. (18) observed that increases in occlusion pressure only altered the RPE response in BFR protocols that used an exercising load of $20 \% 1 \mathrm{RM}$, with no significant effect at $30 \% 1 \mathrm{RM}$. On the contrary, our results showed that increases in occlusion pressure altered the RPE response, even though we used a BFR protocol exercising load of 30\% 1RM. Discrepancies between the studies could be related to different occlusion pressure prescriptions (individualized vs. non-individualized) and/or cuff width [wider (this study: $17.5 \mathrm{~cm}$ ) vs. narrow (18) $5 \mathrm{~cm}$ ] and importantly the use of static BFR pressure applications versus dynamically adjusting BFR pressure application. The use of non-individualized occlusion pressure prescription possibly represents a different stimulus to each subject, affecting their RPE response. For example, Loenneke et al. (17) suggest that the degree of BFR could be influenced by differences between subjects (e.g., leg circumference), as differences in the amount of tissue surrounding the blood vessels could influence the pressure exerted on the vasculature. Thus, using an individualized occlusion pressure is necessary to equate the restrictive stimulus to all subjects. Regarding cuff width, wider cuffs are associated with higher RPE values than narrow ones, at least for non-individualized occlusion pressure prescriptions (i.e., the same occlusion pressure for all participants) (23). Considering that cuff width influences the occlusion pressure necessary to reduce blood flow to muscles (10), subjects exercising with wider cuffs and same absolute occlusion pressures would experience greater BFR, which could increase the RPE response. However, to the best of the authors' knowledge, no study has investigated the effects of 

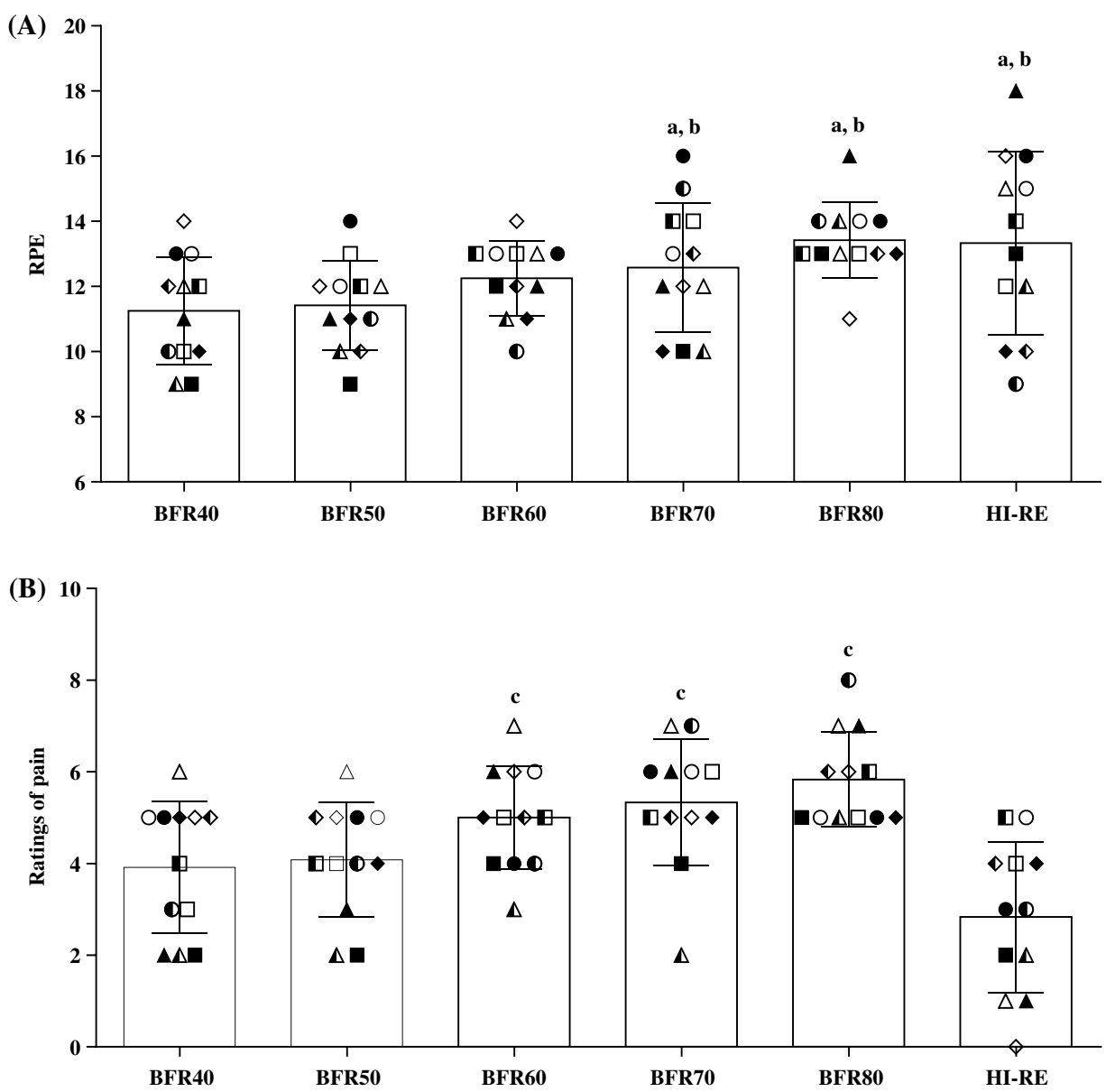

Fig. 1. Mean of all sets of rating of perceived exertion (RPE; A) and ratings of pain (B) for blood-flow restriction (BFR) at 40\% (BFR40), 50\% (BFR50), 60\% (BFR60), 70\% (BFR70), and 80\% (BFR80) of occlusion pressure and high-intensity resistance exercise (HI-RE). Individual responses are represented by different symbols. Values are presented as mean $\pm \mathrm{SD}$. ${ }^{\mathrm{a}}$ Significantly different from BFR40 $(p<0.03)$. ${ }^{\mathrm{b}}$ Significantly different from BFR50 $(p<0.03)$. ${ }^{\mathrm{c}}$ Significantly different from BFR40, BFR50, and HI-RE (all $\left.p<0.009\right)$

occlusion pressure manipulation using different cuff widths on RPE response. Thus, future studies are necessary to understand the relationship between these variables.

Similar to this study, Loenneke et al. (19) investigated the dose response of occlusion pressure (i.e., $40 \%$ up to $90 \%$ of total occlusion restriction) on RPE response. The main results showed that different occlusion pressures did not alter RPE levels. However, these authors failed to equate the RE TTV across the BFR protocols, i.e., some subjects were unable to complete the predetermined number of repetitions. Given that RE TTV can alter the RPE response, these differences preclude an understanding of the effects of occlusion pressure manipulation on RPE parameters. Thus, as this study equated the RE volume across protocols, we can ascertain that modulations in BFR are capable of altering the RPE response during BFR exercise. Furthermore, it has been recently demonstrated that increases in occlusion pressure resulted in elevated RPE responses, even though the higher-pressure 
protocols performed a lower RE volume (20). On the whole, occlusion pressure manipulation seems to have an important impact on the RPE response during BFR protocols.

A secondary purpose of this study was to compare BFR protocols to conventional HI-RE, which is considered as a gold standard measure for increases in muscle mass. Importantly, previous studies have observed similar hypertrophic responses between BFR and HI-RE protocols $(7,12,13,15,21,27,28,34)$. When compared to HI-RE, lowerpressure BFR protocols (i.e., BFR40 and BFR50) presented lower RPE values; however, higher-pressure BFRs (i.e., BFR70 and BFR80) showed similar RPE responses. These results are interesting, as previous studies have demonstrated that low-pressure BFR protocols may be all that is required to promote increases in muscle mass $(6,12,13,15)$. For instance, a recent meta-analysis carried out by our group showed that the magnitude of occlusion pressure did not affect muscle hypertrophy (14). Thus, practitioners and coaches can use BFR protocols for individuals who are unable to withstand the high mechanical load placed upon the joints, typically associated in HI-RE training programs.

With regard to the ratings of pain, our results demonstrated that BFR affected the pain response, corroborating with other studies $(16,30)$. Even more interestingly, pain response was affected by occlusion pressure manipulation, with higher pain levels in the higherpressure BFR protocols. Similarly, Counts et al. (6) observed higher levels of discomfort with higher occlusion pressure BFR protocols ( $40 \%$ vs. $90 \%$ of complete occlusion restriction). When compared to HI-RE, our results showed similar pain levels compared to lower-pressure BFR protocols (i.e., BFR40 and BFR50). On the other hand, higher occlusion pressures applied on the lower body promote values of pain even higher than HI-RE. Collectively, our results suggest that higher occlusion pressures (BFR60, BFR70, and BFR80) during BFR protocols can be even more uncomfortable than conventional RE programs.

Considering that TTV may affect our dependent variables, we opted to equalize TTV to avoid a possible bias and isolate the variable "occlusion pressure," whereas we do not know how different TTVs would affect the measured variable. Some factors may partly explain the successful work that matched TTV, such as: (1) the use of a static BFR pressure control, (2) a lower volume in BFR-RE (i.e., $3 \times 15$ repetition) compared to other BFR-RE studies $(1 \times 30$ reps $+3 \times 15$ reps $)$, and $(3)$ the familiarization sessions with BFR.

In conclusion, low-pressure BFR protocols (BFR40 and BFR50) promote lower RPE and pain compared to high BFR protocols (between $60 \%$ and $80 \%$ of occlusion pressure) when TTV is equalized. In addition, low-pressure BFR promotes lower RPE, but similar levels of pain than HI-RE.

\section{Acknowledgements}

The authors are grateful to Fundação de Amparo à Pesquisa do Estado de São Paulo (FAPESP) - process number: 2015/05660-4.

\section{Conflict of interest}

The authors reported no potential conflict of interest.

\section{REFERENCES}

1. Abbiss CR, Peiffer JJ, Meeusen R, Skorski S: Role of ratings of perceived exertion during self-paced exercise: what are we actually measuring? Sports Med. 45, 1235-1243 (2015) 
2. ACSM: American College of Sports Medicine position stand. Progression models in resistance training for healthy adults. Med. Sci. Sports Exerc. 41, 687-708 (2009)

3. ACSM: American College of Sports Medicine position stand. Quantity and quality of exercise for developing and maintaining cardiorespiratory, musculoskeletal, and neuromotor fitness in apparently healthy adults: guidance for prescribing exercise. Med. Sci. Sports Exerc. 43, 1334-1359 (2011)

4. Assumpção A, Matsutani LA, Yuan SL, Santo AS, Sauer J, Mango P, Marques AP: Muscle stretching exercises and resistance training in fibromyalgia: which is better? A three-arm randomized controlled trial. Eur. J. Phys. Rehabil Med. 54(5), 663-670 (2018)

5. Brown LE, Weir JP: ASEP procedures recommendation I: accurate assessment of muscular strength and power. J. Exerc. Physiol. 4, 1-21 (2001)

6. Counts BR, Dankel SJ, Barnett BE, Kim D, Mouser JG, Allen KM, Thiebaud RS, Abe T, Bemben MG, Loenneke JP: Influence of relative blood flow restriction pressure on muscle activation and muscle adaptation. Muscle Nerve 53, 438-445 (2016)

7. Ellefsen S, Hammarstrom D, Strand TA, Zacharoff E, Whist JE, Rauk I, Nygaard H, Vegge G, Hanestadhaugen M, Wernbom M, Cumming KT, Ronning R, Raastad T, Ronnestad BR: Blood flow-restricted strength training displays high functional and biological efficacy in women: a within-subject comparison with high-load strength training. Am. J. Physiol. Regul. Integr. Comp. Physiol. 309, R767-R779 (2015)

8. Genner KM, Weston M: A comparison of workload quantification methods in relation to physiological responses to resistance exercise. J. Strength Cond. Res. 28, 2621-2627 (2014)

9. Gualano B, Ugrinowitsch C, Neves M, Jr, Lima FR, Pinto AL, Laurentino G, Tricoli VA, Lancha AH, Jr, Roschel H: Vascular occlusion training for inclusion body myositis: a novel therapeutic approach. J. Vis. Exp. 5, $1-2(2010)$

10. Jessee MB, Buckner SL, Dankel SJ, Counts BR, Abe T, Loenneke JP: The influence of cuff width, sex, and race on arterial occlusion: implications for blood flow restriction research. Sports Med. 46, 913-921 (2016)

11. Johnson DE: Crossover experiments. Wiley Interdiscip. Rev. Comput. Stat. 2, 620-625 (2010)

12. Laurentino GC, Ugrinowitsch C, Roschel H, Aoki MS, Soares AG, Neves M, Jr, Aihara AY, Fernandes Ada R, Tricoli V: Strength training with blood flow restriction diminishes myostatin gene expression. Med. Sci. Sports Exerc. 44, 406-412 (2012)

13. Libardi CA, Chacon-Mikahil MP, Cavaglieri CR, Tricoli V, Roschel H, Vechin FC, Conceicao MS, Ugrinowitsch C: Effect of concurrent training with blood flow restriction in the elderly. Int. J. Sports Med. 36, 395-399 (2015)

14. Lixandrao ME, Ugrinowitsch C, Berton R, Vechin FC, Conceicao MS, Damas F, Libardi CA, Roschel H: Magnitude of muscle strength and mass adaptations between high-load resistance training versus low-load resistance training associated with blood-flow restriction: a systematic review and meta-analysis. Sports Med. 48, 361-378 (2018)

15. Lixandrao ME, Ugrinowitsch C, Laurentino G, Libardi CA, Aihara AY, Cardoso FN, Tricoli V, Roschel H: Effects of exercise intensity and occlusion pressure after 12 weeks of resistance training with blood-flow restriction. Eur. J. Appl. Physiol. 115, 2471-2480 (2015)

16. Loenneke JP, Balapur A, Thrower AD, Barnes JT, Pujol TJ: The perceptual responses to occluded exercise. Int. J. Sports Med. 32, 181-184 (2011)

17. Loenneke JP, Fahs CA, Rossow LM, Sherk VD, Thiebaud RS, Abe T, Bemben DA, Bemben MG: Effects of cuff width on arterial occlusion: implications for blood flow restricted exercise. Eur. J. Appl. Physiol. 112, 29032912 (2012)

18. Loenneke JP, Kim D, Fahs CA, Thiebaud RS, Abe T, Larson RD, Bemben DA, Bemben MG: The effects of resistance exercise with and without different degrees of blood-flow restriction on perceptual responses. J. Sports Sci. 33, 1472-1479 (2015)

19. Loenneke JP, Kim D, Mouser JG, Allen KM, Thiebaud RS, Abe T, Bemben MG: Are there perceptual differences to varying levels of blood flow restriction? Physiol. Behav. 157, 277-280 (2016)

20. Mattocks KT, Jessee MB, Counts BR, Buckner SL, Grant Mouser J, Dankel SJ, Laurentino GC, Loenneke JP: The effects of upper body exercise across different levels of blood flow restriction on arterial occlusion pressure and perceptual responses. Physiol. Behav. 171, 181-186 (2017)

21. Ozaki H, Loenneke JP, Thiebaud RS, Stager JM, Abe T: Possibility of leg muscle hypertrophy by ambulation in older adults: a brief review. Clin. Interv. Aging 8, 369-375 (2013)

22. Poton R, Polito MD: Hemodynamic response to resistance exercise with and without blood flow restriction in healthy subjects. Clin. Physiol. Funct. Imaging 36, 231-236 (2016) 
23. Rossow LM, Fahs CA, Loenneke JP, Thiebaud RS, Sherk VD, Abe T, Bemben MG: Cardiovascular and perceptual responses to blood-flow-restricted resistance exercise with differing restrictive cuffs. Clin. Physiol. Funct. Imaging 32, 331-337 (2012)

24. Row BS, Knutzen KM, Skogsberg NJ: Regulating explosive resistance training intensity using the rating of perceived exertion. J. Strength Cond. Res. 26, 664-671 (2012)

25. Scott BR, Duthie GM, Thornton HR, Dascombe BJ: Training monitoring for resistance exercise: Theory and applications. Sports Med. 46, 687-698 (2016)

26. Takarada Y, Sato Y, Ishii N: Effects of resistance exercise combined with vascular occlusion on muscle function in athletes. Eur. J. Appl. Physiol. 86, 308-314 (2002)

27. Thiebaud RS, Yasuda T, Loenneke JP, Abe T: Effects of low-intensity concentric and eccentric exercise combined with blood flow restriction on indices of exercise-induced muscle damage. Interv. Med. Appl. Sci. 5, 53-59 (2013)

28. Vechin FC, Libardi CA, Conceicao MS, Damas FR, Lixandrao ME, Berton RP, Tricoli VA, Roschel HA, Cavaglieri CR, Chacon-Mikahil MP, Ugrinowitsch C: Comparisons between low-intensity resistance training with blood flow restriction and high-intensity resistance training on quadriceps muscle mass and strength in elderly. J. Strength Cond. Res. 29, 1071-1076 (2015)

29. Wernbom M, Augustsson J, Thomee R: Effects of vascular occlusion on muscular endurance in dynamic knee extension exercise at different submaximal loads. J. Strength Cond. Res. 20, 372-377 (2006)

30. Wernbom M, Jarrebring R, Andreasson MA, Augustsson J: Acute effects of blood flow restriction on muscle activity and endurance during fatiguing dynamic knee extensions at low load. J. Strength Cond. Res. 23, 23892395 (2009)

31. Yasuda T, Brechue WF, Fujita T, Shirakawa J, Sato Y, Abe T: Muscle activation during low-intensity muscle contractions with restricted blood flow. J. Sports Sci. 27, 479-489 (2009)

32. Yasuda T, Fukumura K, Fukuda T, Uchida Y, Iida H, Meguro M, Sato Y, Yamasoba T, Nakajima T: Muscle size and arterial stiffness after blood flow-restricted low-intensity resistance training in older adults. Scand. J. Med. Sci. Sports 24, 799-806 (2014)

33. Yasuda T, Loenneke JP, Thiebaud RS, Abe T: Effects of blood flow restricted low-intensity concentric or eccentric training on muscle size and strength. PLoS One 7, e52843 (2012)

34. Yasuda T, Ogasawara R, Sakamaki M, Ozaki H, Sato Y, Abe T: Combined effects of low-intensity blood flow restriction training and high-intensity resistance training on muscle strength and size. Eur. J. Appl. Physiol. 111, $2525-2533(2011)$ 